\title{
La modélisation d'accompagnement pour une gestion concertée des ressources renouvelables en Thaillande
}

Companion modelling for cooperative management of renewable resources in Thailand

Cécile Barnaud, Guy Trébuil, Panomsak Promburom et François Bousquet

\section{(2) OpenEdition}

Journals

Édition électronique

URL : http://journals.openedition.org/economierurale/512

DOI : 10.4000/economierurale.512

ISSN : 2105-2581

Éditeur

Société Française d'Économie Rurale (SFER)

Édition imprimée

Date de publication : 1 juin 2008

Pagination : 39-59

ISSN : 0013-0559

Référence électronique

Cécile Barnaud, Guy Trébuil, Panomsak Promburom et François Bousquet, «La modélisation

d'accompagnement pour une gestion concertée des ressources renouvelables en Thaillande », Économie rurale [En ligne], 303-304-305 | Janvier-juin 2008, mis en ligne le 30 juin 2010, consulté le 20 avril 2019. URL : http://journals.openedition.org/economierurale/512 ; DOI : 10.4000/ economierurale.512 


\section{La modélisation d'accompagnement pour une gestion concertée des ressources renouvelables en Thaillande}

Cécile BARNAUD • Laboratoire de géographie comparée des Nords et des Suds, Gecko, Université Paris X-Nanterre, CIRAD, UPR Green, Gestion des ressources renouvelables et environnement, Montpellier et Projet Commod, CU-CIRAD, Université Chulalongkorn, Bangkok, Thaïlande

Guy TRÉBUIL • CIRAD, UPR Green, Gestion des ressources renouvelables et environnement, Montpellier et Projet Commod, CU-Cirad, Université Chulalongkorn, Bangkok, Thaïlande

Panomsak PROMBUROM • Université de Lyon et Multiple Cropping Center, MCC, Faculté d'agriculture, Université de Chiang Maï, Thaïlande

François BOUSQUET • CIRAD, UPR Green, Gestion des ressources renouvelables et environnement, Montpellier et Projet Commod, CU-CIRAD, Université Chulalongkorn, Bangkok, Thaïlande

\section{Introduction}

$\mathbf{L}^{\mathrm{a}}$ a Thaïlande a très activement participé au mouvement de mondialisation des échanges des deux dernières décennies. Elle s'est élevée au rang de pays émergent et de $7^{\mathrm{e}}$ puissance agro-exportatrice, mais avec un coût environnemental et social élevé (Trébuil, 1993). La limite d'expansion des terres agricoles a été atteinte et un mouvement de reforestation amorcé, notamment dans les hautes terres du nord de la Thaïlande où les minorités ethniques sont accusées de dégrader les hauts des bassins versants des grands cours d'eau du pays. Depuis le début des années 1990, les conflits d'usage sur la gestion des ressources se multiplient dans cette région, opposant des acteurs de plus en plus nombreux et différenciés (Rutherford, 2002). Perçues par la majorité thaïe comme une menace pour la sécurité nationale comme pour l'environnement, les communautés montagnardes non thaïes n'ont eu jusqu'à présent que peu de moyens pour défendre leurs intérêts (McKinnon et Vienne, 1989). Cependant, avec la montée en puissance des mouvements civils défendant les droits de ces communautés, le discours officiel évolue. Le processus de démocratisation de la vie politique amorcé dans les années 1990 a abouti à l'adoption en 1997 de la nouvelle «Constitution du Peuple » qui stipule la décentralisation de la gestion des ressources renouvelables et le renforcement du rôle et des moyens des administrations au niveau du sousdistrict (les TAO, Tambon Administrative Organization). De nouvelles opportunités s'offrent aux communautés dont on reconnaît, au moins sur le papier, des droits et des responsabilités (Ganjanapan, 2002). Mais l'impact de cette décentralisation est actuellement limité par le manque de dialogue entre les communautés et les administrations dont les discours évoluent plus vite que les mentalités, le risque étant de voir la décentralisation se solder par une simple déconcentration du pouvoir central (Arghiros, 2001).

Comment la paysannerie pauvre en milieu montagnard pourrait-elle tirer partie de ces récentes transformations institutionnelles ? Comment favoriser une gestion plus concertée des ressources sécurisant cette petite agriculture familiale tout en limitant la dégradation de l'environnement et les inquiétudes des puissants acteurs des plaines? Quel type de recherche pourrait favoriser le dialogue, actuellement limité, entre les communautés et les administra- 
tions locales, clef de voûte du processus de décentralisation?

Pour que la participation des communautés aux prises de décision soit effective, il semble nécessaire non seulement de faire évoluer les administrations, mais également de renforcer la capacité des communautés à maîtriser les enjeux des situations locales complexes, à formuler des propositions adaptées et à les faire entendre auprès des autorités. Les travaux de recherche récents dans le domaine de la gestion des ressources renouvelables soulignent le rôle déterminant de la coordination entre les parties prenantes impliquées à différents niveaux d'organisation pour faciliter l'émergence d'une agriculture à la fois écologiquement viable et socialement équitable (Ostrom et al, 1994 ; Röling et Wagemakers, 1998). Par ailleurs, face à l'incertitude croissante des systèmes, due à leur rapide évolution, d'autres soulignent la nécessité d'une gestion adaptative au moyen d'un apprentissage collectif continu renforçant la capacité d'adaptation des acteurs (Holling, 2001). La démarche de modélisation d'accompagnement $(\text { ComMod })^{1}$ que nous avons testée dans le travail ici présenté repose sur la construction collective, itérative et continue d'une représentation partagée de la situation, support adaptatif d'un processus d'apprentissage collectif et de négociation entre les multiples parties prenantes (Bousquet et al, 1996 ; Commod, 2005).

L'objectif de cet article est d'évaluer les apports et les limites de cette démarche pour faciliter, dans le contexte de la décentralisation, un processus d'apprentissage collectif et de concertation au sein des villages montagnards et avec l'administration locale du TAO, en vue d'une gestion durable des ressources. L'évolution du contexte institutionnel de la gestion des ressources dans cette région sera d'abord retracée afin d'en dégager les enjeux. Les

1. Pour Companion Modelling. principaux apports théoriques auxquels nous nous référons seront ensuite introduits, ainsi que les principes de la démarche de modélisation d'accompagnement. Après avoir présenté la méthodologie adoptée et l'expérience réalisée dans un village akha de la province de Chiang Raï, les résultats et les limites du processus d'apprentissage suscité seront discutés. Enfin, la question du renforcement de l'impact de cette démarche sera abordée en conclusion.

\section{Évolution du contexte institutionnel de gestion des ressources}

Afin d'analyser les apports et les limites de la modélisation d'accompagnement pour faciliter une gestion décentralisée des ressources dans le nord de la Thaïlande, il est nécessaire de présenter le contexte institutionnel, son évolution récente et ses enjeux actuels. À chaque étape de cette évolution, nous soulignerons les répercussions des règles en vigueur sur les conditions socio-économiques de la petite paysannerie montagnarde, ainsi que sur l'état des ressources.

\section{Avant 1980 : des institutions coutumières, une économie d'autosubsistance}

Au royaume du Siam, la gestion des ressources naturelles est traditionnellement une affaire d'État. Dans les montagnes du nord du pays, toutes les terres appartiennent officiellement à la famille royale. Les minorités ethniques y sont tolérées mais, n'ayant pas même le droit de revendiquer la nationalité thaïlandaise, elles n'ont aucun droit officiel sur les terres qu'elles exploitent. Jusqu'aux années 1970 cependant le gouvernement n'accorde que peu d'importance à cette périphérie reculée et n'y exerce aucun contrôle réel. Les règles en vigueur pour la gestion des ressources sont donc les institutions coutumières spécifiques à chaque groupe ethnique et mises en place au niveau de chaque communauté (Goodman, 1996). 
Dans ces communautés relativement isolées domine une économie de subsistance basée sur la production de riz pluvial et de maïs. Chez certaines, dont les Akhas, le pavot à opium est une première culture de rente dont le rapport élevé valeur au poids facilite le transport sur les pistes muletières sillonnant le fameux Triangle d'or. Les ressources en terres, forêts et eau sont encore relativement abondantes. Avec une densité de population inférieure à 20 habitants par $\mathrm{km}^{2}$, les montagnards pratiquent une agriculture sur abattis-brûlis à longues jachères (10 à 20 ans) autorisant le plus souvent le renouvellement du couvert arboré (Rerkasem et al., 1994).

\section{Les années 1980 : une gestion centra- lisée des ressources et l'intégration à l'économie de marché}

Les décennies 1970 et 1980 sont marquées par une politique d'intégration nationale que l'on peut résumer ainsi : "Le gouvernement thaïlandais voudrait bien intégrer les hautes terres, mais il les préférerait inoccupées » (Pungprasert, 1989). La stigmatisation des montagnards par les Thaïs des basses terres véhicule des images de communistes potentiels, de trafiquants de drogue menaçant la sécurité de la nation, ou encore de «tribus » arriérées aux vices multiples qui détruisent les forêts. Cette dernière idée justifie le renforcement d'une gestion des ressources hautement centralisée se soldant par la mise en défend de près d'un tiers de la superficie du pays. Mais l'éloignement de Bangkok, la corruption et le manque d'agents forestiers pour faire appliquer ces lois laissent libre court aux processus de colonisation agricole et de déforestation. Non reconnues par l'État, les règles coutumières locales sont affaiblies et l'accès aux ressources accaparé par les plus influents, ce qui renforce les inégalités (Ganjanapan, 2002). C'est le constat de l'échec de cette gestion centralisée qui justifiera en partie les politiques de décentralisation de la gestion des ressources initiées dans les années 1990, comme nous le verrons dans la section suivante.

Dopée par une croissance économique rapide après 1986, cette période fut également marquée par l'intégration des communautés montagnardes à l'économie de marché. Les deux principaux leviers de cette intégration furent le désenclavement par le réseau routier et la multiplication des grands projets de développement visant à remplacer l'abattis-brûlis par des cultures permanentes ainsi qu'à substituer des cultures commerciales horticoles à la production d'opium ${ }^{2}$ (McKinnon et Vienne, op. cit. ; Le Meur, 2000). Cette transition agraire est à l'origine d'une différenciation socio-économique marginalisant une large frange de la paysannerie montagnarde aux prises avec des incertitudes croissantes (insécurité foncière, fluctuations des prix, endettement, etc.). Par ailleurs, face aux fortes incitations à produire (accroissement démographique, demande des marchés), les ressources renouvelables des hautes terres (déjà largement entamées par l'exploitation commerciale du bois depuis les années 1960) sont encore fortement dégradées au cours de cette décennie (déforestation, érosion des sols, dégradation des hydrosystèmes, pertes de biodiversité, etc.) (Trébuil, op. cit. ; Thomas et al, 2002 ; Rola et Coxhead, 2005).

\section{Les années 1990 : participation et environnement, duel ou duo ?}

C'est dans les années 1990 qu'entrent en scène deux enjeux brûlants de l'actualité thaillandaise :

- les préoccupations environnementales faisant suite à une prise de conscience des externalités négatives de la croissance économique ;

2. Mais selon certains auteurs, l'objectif officieux de ces interventions a toujours été de fixer les montagnards afin de mieux les contrôler (McKinnon et Vienne, op. cit.) 
- la nouvelle politique de décentralisation issue du processus de démocratisation de la vie politique ayant été permis par l'émergence d'une classe moyenne et la montée en puissance d'une société civile.

Deux façons de penser l'articulation entre ces deux enjeux dans le contexte du nord de la Thaïlande se distinguent, correspondant à deux courants de pensées opposés.

D'un côté, les environnementalistes tiennent les montagnards pour responsables de la dégradation du milieu et préconisent de limiter leur accès aux ressources. C'est ainsi que l'on a vu le nombre de parcs nationaux augmenter récemment dans le nord du pays.

D'un autre côté, les défenseurs d'une gestion communautaire des ressources clament que les communautés sont les plus à même de gérer durablement des ressources dont leur survie dépend. Il s'agit généralement d'organisations non gouvernementales (ONG) dont les actions, nombreuses et souvent réussies, gardent cependant un impact limité car elles ne sont pas relayées par les organismes gouvernementaux aux niveaux supérieurs. Ces ONG sont généralement en conflit avec les administrations locales, qu'elles perçoivent comme des «ennemis » des communautés. Or la décentralisation a mis en place, au moins sur le papier, des institutions au service d'une plus grande participation des communautés dans la gestion des ressources, notamment les administrations du sous-district (les TAO). La nouvelle loi de 1994 sur les TAO augmente les responsabilités et les moyens qui leur sont octroyés et instaure un conseil composé de deux représentants par village, directement élus par les villageois.

Dans ce contexte, notre recherche a été axée sur l'évaluation des apports et des limites de ComMod en tant que nouvelle forme de communication et de coordination entre l'échelon villageois et les organisations gouvernementales (notamment les TAO) afin que la décentralisation soit porteuse d'une gestion plus concertée des ressources locales.

\section{Cadre conceptuel pour une gestion concertée des ressources}

\section{Institutions et coordination}

Les travaux d'Ostrom (op. cit.) nous renseignent sur les conditions institutionnelles favorables à une gestion durable des ressources renouvelables communes. Cette chercheuse s'est opposée à la théorie de la «tragédie des communs » formulée par Hardin (1968), lequel voit les ressources communes «en accès libre » vouées à disparaître car nécessairement surexploitées par ses utilisateurs dont l'intérêt individuel n'est pas d'économiser la ressource si les autres utilisateurs ne font pas de même. Selon lui, seules la gestion centralisée par l'État ou la propriété privée sont à même de garantir le renouvellement des ressources, idée qui influence encore les politiques environnementales thaïlandaises. L'une des failles du raisonnement de Hardin selon Ostrom est d'avoir négligé l'importance des institutions coutumières qui font que dans de nombreuses situations, l'accès aux ressources communes n'est pas libre mais régulé par un ensemble de règles reconnues par les utilisateurs. Cette erreur est fondée sur l'une des acceptions classiques du terme institution qui n'inclut que les organisations officielles de l'État. Selon Ostrom, une institution est un ensemble de règles en vigueur, de «prescriptions qui déterminent si telle ou telle action (ou résultat) est requise, interdite, ou permise, et quelles seront les sanctions en cas d'infraction $\gg^{3}$. En combinant des analyses théoriques et empiriques, Ostrom (2005) identifie un certain nombre de principes permettant d'échapper à la tragédie des communs.

3. Cf. Ostrom, 1994, page 38. 
- Accès aux ressources clairement définies (qui, quand, comment, obligations, sanctions, gestion des règles) ;

- Des règles définies collectivement et renforcées par des relations de confiance, et définies par les utilisateurs eux-mêmes ; - En lien avec les instances institutionnelles supérieures,

- Des règles évolutives.

Ainsi, la coordination des multiples parties prenantes à différents niveaux d'organisation est un facteur clef de l'émergence d'institutions favorables à une gestion durable des ressources. Berkes et al. (1991) ont défini la cogestion des ressources comme un partage des pouvoirs et des responsabilités entre les gouvernements et les usagers locaux des ressources. Carlsson et Berkes (2004) ajoutent que ni les gouvernements ni les communautés locales ne sont des structures monolithiques et homogènes, et que la cogestion doit donc être pensée comme un processus continu d'apprentissage collectif et de négociation en vue d'une recherche de solutions au sein de réseaux sociaux.

\section{Apprentissage collectif et négociation}

Mais comment ces notions d'apprentissage collectif et de négociation s'articulent-elles avec celle de gestion des ressources renouvelables?

Étant donné l'incertitude croissante et l'évolution permanente des systèmes agraires, tout particulièrement en Asie, il est vain de chercher à établir définitivement quelles sont les règles les plus favorables à une gestion durable des ressources. Il est préférable de chercher à améliorer la qualité des processus de décision menant à la formulation de ces règles (Funtowicz et Ravetz, 1994). Röling et Wagemakers (op. cit.) se sont intéressés à la qualité de ces processus de décision dans le domaine agricole. Ils définissent la durabilité de l'agriculture comme une propriété du système agraire émergeant de la coordi- nation entre les multiples utilisateurs et gestionnaires des ressources. Ils font l'hypothèse que les perceptions qu'ont les gens de leur environnement (écologique et social) déterminent leurs actions sur et dans cet environnement, ces perceptions pouvant être modifiées par une meilleure compréhension de cet environnement et par les interactions avec les autres parties prenantes. De ces modifications de perception naît l'apprentissage. C'est donc par un processus évolutif et continu d'apprentissage collectif que des acteurs créent les conditions d'émergence d'une agriculture durable.

Mais la notion d'apprentissage collectif ne peut nier l'existence de divergences d'intérêts entre les parties prenantes d'un système agraire à la paysannerie différenciée, reproche souvent fait aux approches participatives, notamment au nord de la Thaïlande où certains auteurs ont pu parler d' « ethno-romantisme » (Neef, 2004). Nombreuses sont les situations où le consensus est difficile, voire impossible à établir, d'où l'importance de la mise en place de processus de négociation (Leeuwis et Van Den Ban, 2004).

\section{La modélisation d'accompagnement}

La démarche ComMod a pour objectif de faciliter de tels processus d'apprentissage collectif et de négociation à l'échelle de communautés villageoises dans le domaine de la gestion des ressources renouvelables (Bousquet et al, 1996 ; Commod, 2005). Développée depuis une dizaine d'années, elle a été testée dans des contextes géographiques et à propos de thématiques variés (D'Aquino et al., 2003 ; Étienne, 2003 ; Bousquet et al, 2005 ; Gurung et al, 2006). Ses principes sont proches de ceux de la démarche de gestion patrimoniale dans laquelle la résolution de problèmes multi-acteurs repose sur la négociation d'objectifs communs à long terme et l'identification et l'évaluation de scénarios à mettre en œuvre pour 
les atteindre (Ollagnon, 1989). L'hypothèse est faite que s'il est difficile à des acteurs aux intérêts divergents de s'accorder sur des actions à court terme, il peut être plus aisé de les faire s'entendre d'abord sur des objectifs à plus long terme.

La démarche ComMod propose la construction collective, itérative et continue d'une représentation commune du système à gérer, support adaptif d'un processus d'apprentissage collectif et de négociation. L'une des exigences clef de cette démarche est l'explicitation et la confrontation systématiques des hypothèses des chercheurs auprès des parties prenantes concernées, ce qui impose une pratique itérative dans laquelle des activités de modélisation et de terrain alternent et s'enrichissent mutuellement ${ }^{4}$ (Commod, 2005). Les modèles sont ainsi conçus et utilisés selon un processus cyclique, chaque cycle étant composé des trois phases suivantes :

- synthèse des données secondaires et études de terrain complémentaires pour analyser une situation à propos d'un problème donné de gestion des ressources ;

- « conception d'un modèle à partir de la représentation de la situation issue du travail d'analyse-diagnostic préalable ;

- « simulations à l' aide du modèle avec les acteurs locaux afin de confronter la représentation initiale des chercheurs à celle des acteurs locaux et de faciliter un processus de concertation entre eux.

Cette dernière phase peut mener les chercheurs à la mise un œuvre d'un nouveau cycle ComMod pour adapter le modèle aux représentations et aux préoccupations des acteurs locaux, lesquelles peuvent évoluer au cours du processus d'apprentissage collectif suscité.

4. Les fondements de la démarche ComMod et de son utilisation ont été publiés dans une charte (Commod, 2005).
Les outils de modélisation et de simulation privilégiés par ComMod à ce jour sont les systèmes multi-agents (SMA) et les jeux de rôles. Les SMA correspondent à une famille de modèles informatiques issue du domaine de l'intelligence artificielle distribuée. Ferber (1999) définit un système multi-agents comme un ensemble d'entités autonomes en interaction ; ces entités autonomes - les agents - situées dans un environnement commun sont douées d'un objectif et de représentations partielles de leur environnement. Les SMA visent à appréhender les effets des interactions et de la coordination entre ces processus autonomes, desquels peut résulter une dynamique collective nommée émergence. Les SMA sont adaptés aux problèmes de gestion des ressources car ils représentent et simulent les interactions entre des agents sociaux hétérogènes, ainsi qu'entre ces agents et leur environnement, lequel est doté de sa propre dynamique écologique (Bousquet et al, 1993 ; Lansing et Kremer, 1993). Les jeux de rôles sont des modèles (au sens large) permettant la mise en situation de joueurs, à l'aide de différents supports (cartes, argent, etc.) et sont aussi des dispositifs les amènant à prendre des décisions d'action répétées dans le temps en fonction du rôle qui leur est assigné, des contraintes qui leur sont imposées, et des interactions avec leur environnement physique et social. Cette mise en situation hypothétique permet de mieux comprendre les motivations qui justifient les comportements, et de discuter du lien existant entre le modèle joué et la réalité. Par sa mise en situation distanciée de la réalité, le jeu stimule des échanges entre les différents acteurs du système et peut ouvrir des voies encore inexplorées de négociation et d'identification de solutions aux problèmes rencontrés (Mermet, 1993). Dans la démarche ComMod, le jeu de rôles est souvent conçu comme une version simplifiée du modèle SMA. Il 
permet ainsi « d'ouvrir la boîte noire du modèle » et offre aux acteurs locaux l'opportunité de critiquer la représentation du problème proposée par les chercheurs (Barreteau et al, 2001). Cependant, la mise en œuvre d'une session de jeu est longue, coûteuse et contraignante ; le nombre de scénarios que l'on peut tester avec cet outil est donc limité. Le modèle SMA validé par les acteurs locaux permet de lever cet obstacle car il permet de stimuler rapidement et à long terme de nombreux scénarios.

C'est cette démarche et cette association d'outils que nous avons testées pour faciliter la gestion concertée de l'usage des terres agricoles du village Akha de Mae Salaep, en interaction avec son TAO, dans la province de Chiang Raï.

\section{Modélisation d'accompagnement à Mae Salaep}

\section{Situation agraire locale}

Le village de Mae Salaep est situé à $700 \mathrm{~m}$ d'altitude, à une journée de marche de la frontière birmane. Ses habitants, d'origine akha, s'y sont installés au cours du siècle dernier au gré de vagues migrations successives pour fuir la guerre civile en Birmanie. Depuis le début des années 1980, sous les effets combinés de l'intégration à l'économie de marché, des politiques environnementales et de la pression démographique croissante, leur système agraire de subsistance basé sur l'abattis-brûlis a laissé place à une agriculture commerciale et quasi permanente (Trébuil et al, 1997 ; Trébuil et al, 2000). Les puissants acteurs des plaines perçoivent ces modifications de pratiques comme un accroissement des risques d'érosion sur les pentes des hauts de bassins versants et redoutent les inondations et la sédimentation dans leurs réseaux d'irrigation. Pour les habitants de Mae Salaep, le problème d'érosion est avant tout le risque de voir les agents du Département royal des forêts venir confisquer leurs terres. À Mae Salaep comme dans le nord du pays, les multiples tentatives d'introduction de mesures de conservation des sols (essentiellement des bandes enherbées et des haies arbustives le long des courbes de niveau) dans les années 1980 et au début des années 1990 ont été peu fructueuses, ces propositions n'étant pas adaptées aux conditions socio-économiques locales. Dans le contexte de « révolution horticole » qui préside actuellement dans cette région, l'adoption de cultures commerciales pérennes est vue par les villageois comme une solution prometteuse. Par rapport aux cultures annuelles dont la couverture du sol est temporaire, ces cultures pérennes par leur couvert permanent limitent les risques d'érosion (à condition que le couvert herbacé sous canopée soit conservé) et permettent d'éloigner la menace d'éviction par les autorités. Elles offrent des revenus agricoles plus élevés que les cultures annuelles à faible valeur marchande. Moins exigeantes en maind'œuvre, elles laissent davantage de temps pour l'emploi non agricole qui fournit plus de la moitié des revenus dans le village.

Les plantations de litchi dans le village remontent au début des années 1990. Si ces plantations sont irriguées, les rendements sont stabilisés et peuvent être décuplés. Introduit peu après les litchis, le thé vert Assam a une plus faible valeur ajoutée, mais est produit à moindre risque en culture non irriguée et offre un revenu plus stable et plus régulier que les litchis. Il fait figure de «culture pérenne des pauvres » bien qu'il soit encore inaccessible aux petites exploitations du village. Récemment, le gouvernement a introduit une variété de thé vert Oolong à valeur commerciale élevée mais dont la culture nécessite un important recours aux intrants et à l'irrigation. De plus, tous les agriculteurs n'ont pas les moyens d'investir dans de telles plantations. On 
Tableau 1. Différenciation socio-économique entre exploitations agricoles à Mae Salaep dans la province de Chiang Raï au nord de la Thaillande (2005)

\begin{tabular}{l|l|l|l}
\hline Type & $\begin{array}{l}\text { A. Petites } \\
\text { exploitations } \\
\text { vulnérables }\end{array}$ & $\begin{array}{l}\text { B. Exploitations } \\
\text { auto-suffisantes de taille } \\
\text { de taille moyenne }\end{array}$ & $\begin{array}{l}\text { C. Exploitations marchandes } \\
\text { et diversifiées }\end{array}$ \\
\hline Superficie (ha/UTH *) & $0,4-0,8$ & $0,8-2,4$ & $1,3-3,2$ \\
\hline $\begin{array}{l}\text { Principaux systèmes } \\
\text { de culture }\end{array}$ & Maïs pluvial & $\begin{array}{l}\text { Riz pluvial, maïs, petites } \\
\text { plantations non irriguées } \\
\text { de thé Assam ou de litchi }\end{array}$ & $\begin{array}{l}\text { Riz inondé, maïs, } \\
\text { plantations irriguées } \\
\text { de litchi et/ou de thé Oolong }\end{array}$ \\
\hline Activité non agricole & Pour survivre & Pour sécuriser l'exploitation & Pour investir \\
\hline $\begin{array}{l}\text { Revenu total familial } \\
\text { moyen (euros/an)** }\end{array}$ & 240 & 500 & 1400 \\
\hline $\begin{array}{l}\text { Capacité } \\
\text { d'investissement }\end{array}$ & Nulle & Faible & Importante \\
\hline
\end{tabular}

* Unité Travail Homme équivalent 300 jours de travail par an.

** Seuil de survie dans le village : 250 euros/famille/an, salaire ouvrier dans les plaines : 900 euros/an.

Source : les auteurs

distingue trois grands types d'exploitations (tableau 1) :

- les petites exploitations de type A ne cultivent que du maïs « pour acheter du riz » et qui ne peuvent survivre sans les revenus complémentaires du travail de journaliers agricoles, mais qui commencent à investir dans de petites plantations de thé Assam; - les exploitations moyennes de type B aux revenus agricoles suffisants pour assurer les besoins de subsistance de la famille et qui ont souvent investi dans des petites plantations non irriguées de thé Assam ou de litchis ;

- les exploitations de type $\mathrm{C}$, marchandes et diversifiées, disposants d'opportunités de travail hors exploitation rentables et qui investissent dans de grandes plantations irriguées de litchis et parfois de thé Oolong.

\section{Enjeux de la situation agraire locale au cours des trois cycles ComMod}

L'objectif du processus ComMod n'est pas de fournir des solutions aux acteurs locaux, mais de leur proposer une démarche et des outils leur permettant de mieux comprendre par eux-mêmes leurs problèmes et de réfléchir collectivement à de possibles solutions. Dans le processus d'apprentissage collectif suscité et accom- pagné, l'analyse d'un problème au cours d'un cycle peut amener les acteurs locaux à soulever de nouveaux problèmes. C'est ainsi que les problèmes d'érosion des sols, d'inégal accès aux cultures pérennes et à l'eau d'irrigation ont successivement été soulevés au cours des trois cycles ComMod développés avec les villageois, les outils mobilisés étant à chaque cycle adaptés aux évolutions de leurs préoccupations (figure 1). Cet article analyse en détails les résultats du troisième cycle centré sur le problème du partage de l'eau. Néanmoins, il est important de comprendre le contexte dans lequel cette question a émergé.

\section{Les deux premiers cycles ComMod}

$\mathrm{Au}$ cours du premier cycle, le point focal des discussions évolua des aspects agroécologiques de l'érosion des terres vers la solution envisagée : l'adoption de cultures pérennes (Trébuil et al, 2002). Les participants demandèrent alors de modifier le modèle pour aborder les conditions socio-économiques de leur adoption (figure 2). Ce fut l'objet d'un second cycle portant sur les interactions entre crédit formel et informel, travail hors exploitation et investissement dans les plantations (Barnaud et al, 2006a). 
RECHERCHES

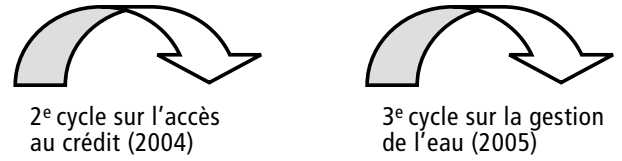

1 er cycle sur l'érosion au crédit (2004) de l'eau (2005)

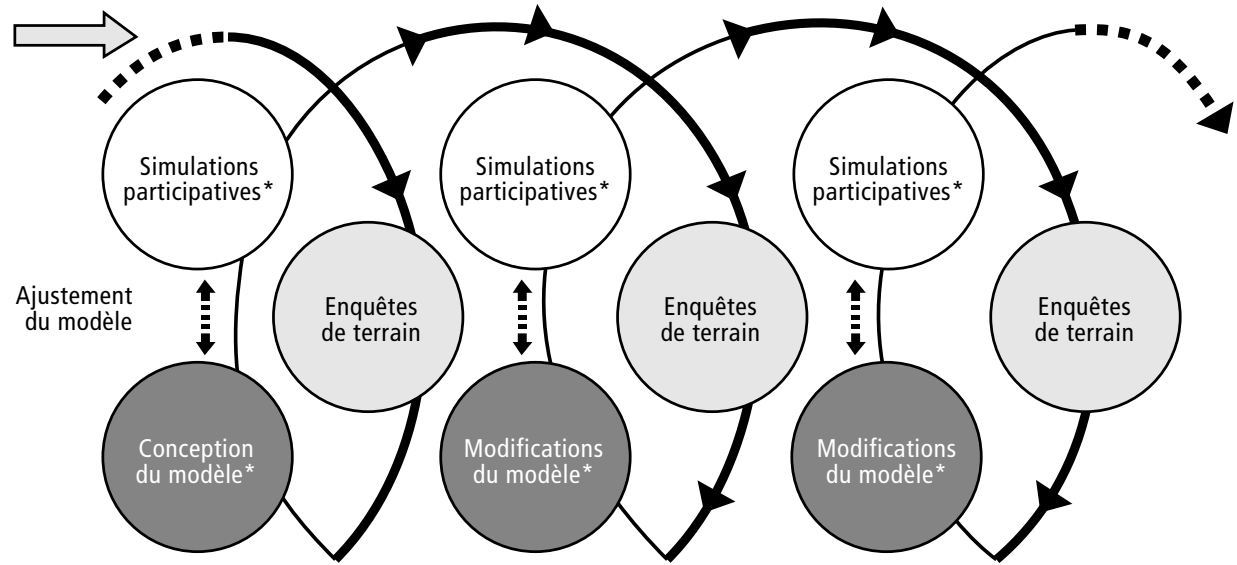

* 1 modèle conceptuel sous 2 formes : jeu de rôles et système multi-agent

Source : conception des auteurs

Figure 2. Évolution des interactions clés discutées au cours des trois cycles ComMod conduits à Mae Salaep entre 2002 et 2005

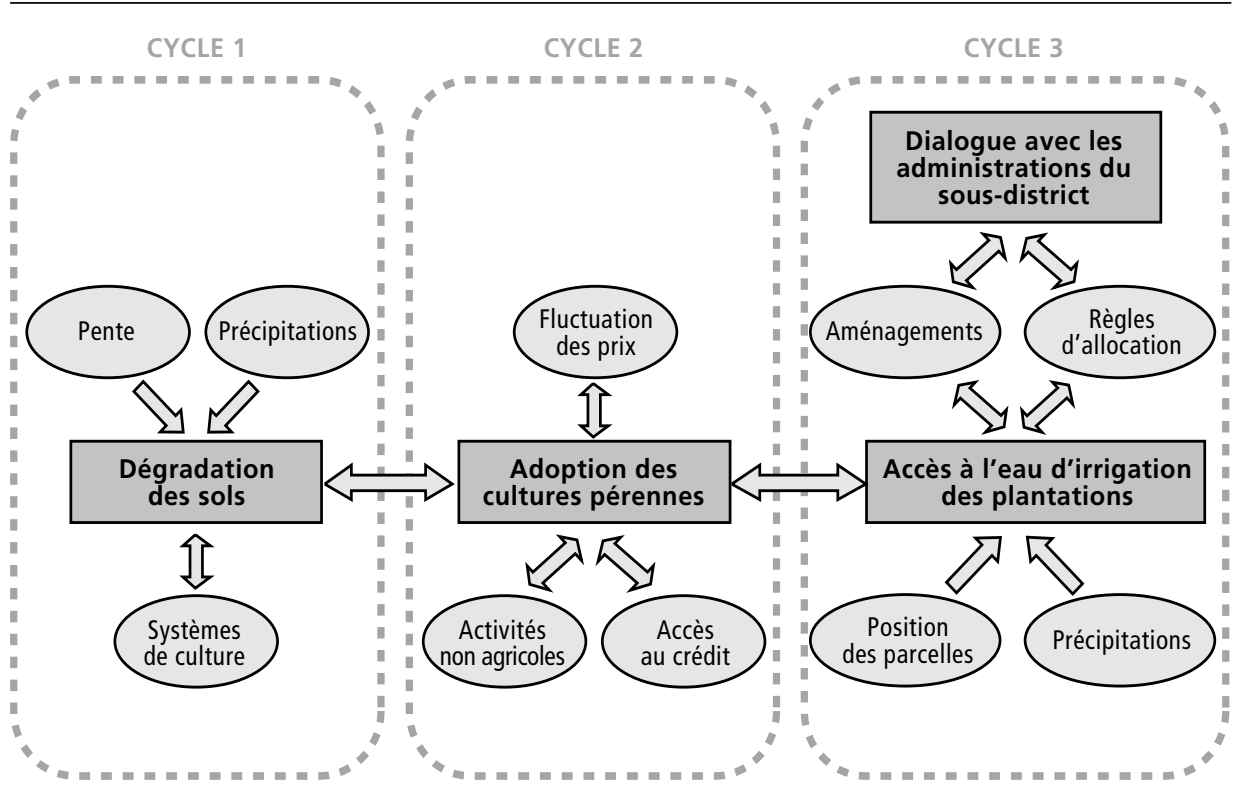


Les participants formulèrent des scénarios de changement de règles de crédit pour pallier le problème d'inégal accès aux cultures pérennes. L'une des propositions consistait à augmenter la durée des prêts alloués par le gouvernement dans le cadre d'une politique de crédit rural décentralisé. Mais c'est au niveau du gouvernement que de telles décisions se prennent. Les villageois expliquèrent alors que plusieurs villages avaient déjà alerté le gouvernement de cette proposition (indépendamment du processus ComMod), et que si le gouvernement l'acceptait, de nouvelles sessions de jeu seraient utiles pour leur permettre de s'adapter collectivement à ces changements. Mais le gouvernement fut renversé depuis, cette proposition ne put donc être mise en place. Ceci illustre d'ailleurs les limites de cette politique de crédit rural dite décentralisée (Barnaud et al, 2007). Malgré cela, les participants retenaient de cette expérience les bénéfices de la concertation entre villageois pour penser ensemble des projets d'avenir ainsi que la nécessité d'un soutien institutionnel pour leur mise en œuvre. Lors des enquêtes d'évaluation de ce second cycle, les villageois émirent deux souhaits pour la suite : introduire la question de l'eau d'irrigation dans le jeu et faire participer des représentants du TAO «pour qu'ils sachent ce qui se passe dans le village ». Le troisième cycle permettait donc d'aborder la concertation inter-institutionnelle entre village et sous-district.

\section{Troisième cycle ComMod : gestion de l'eau et dialogue avec le TAO}

L'irrigation gravitaire au moyen de canalisations captant l'eau des ruisseaux est apparue au début des années 1990 avec l'introduction des vergers de litchis dont les rendements sont stabilisés et fortement augmentés par une irrigation lors de la floraison en saison sèche. Chaque ruisseau ne permettant d'irriguer que deux ou trois exploitations, la règle adoptée fut celle du «premier arrivé, premier servi » : si un agriculteur a installé sa prise d'eau sur un ruisseau, aucun autre ne peut venir ensuite placer la sienne en amont. Mais l'augmentation du nombre d'exploitants prétendant à l'irrigation créa des tensions.

Les villageois lient la question de l'eau à celle de la participation du TAO au processus ComMod car ce dernier peut financer des projets d'aménagement hydroagricoles. Chaque village peut demander un tel soutien financier par ses représentants élus siégeant au conseil du TAO. Mais les représentants villageois rencontrés décrivent encore leur tâche comme un transfert d'informations depuis les administrations vers les villages et non l'inverse. Quant aux villageois, si la plupart se disent peu concernés, d'autres, suffisamment influents, se plaignent de l'inadéquation entre les demandes villageoises et les projets mis en place. La répartition des bénéfices des projets est également source de mécontentement, les représentants villageois étant parfois accusés de favoriser leur clan qui est aussi leur principal électorat. Le troisième cycle ComMod visait donc à stimuler un processus d'apprentissage collectif sur la gestion de l'eau dans le sous bassin-versant entre les villageois et avec le TAO.

\section{Méthode et outils mis en œuvre dans le troisième cycle ComMod}

\section{Enquêtes de terrain et conception du jeu}

de rôles et du modèle SMA

Ce troisième cycle ComMod démarra par une série d'entretiens auprès des villageois et des représentants du TAO pour mieux comprendre le problème du partage de l'eau d'irrigation dans le village ainsi que le fonctionnement du TAO. Notre compréhension de la gestion de l'eau a été formalisée sous forme d'un modèle conceptuel utilisé pour modifier le jeu de rôles et le modèle SMA existants (Barnaud et al, 2008) afin qu'ils puissent servir pour discuter du problème de gestion de l'eau. Les principes du jeu de 


\section{Encadré 1. Les principales règles du jeu de rôles du troisième cycle ComMod à Mae Salaep}

Les 12 villageois-participants jouent le rôle de chefs de famille gérant différents types d'exploitations familiales (type A, B ou C) caractérisées par des quantités données de ressources en terre, en travail et en capital. Leurs parcelles sont localisées sur un plateau de jeu en trois dimensions représentant un bassin versant composé de plusieurs sousunités. Chaque année, les joueurs vont successivement :

- essayer, s'ils le souhaitent, d'obtenir un crédit au « bureau du crédit »;

- décider d'envoyer (ou pas) des membres de leur famille travailler hors exploitation,

- allouer des cultures à leurs parcelles (en fonction de leur localisation, de la force de travail et de la trésorerie disponibles) ;

- décider d'investir (ou pas) dans des installations d'irrigation ;

- aller solder leurs comptes au «marché » (en prenant en compte les besoins pour la consommation familiale) ;

- rembourser si besoin leur crédit.

La situation au début du jeu correspond à celle du village il y a une quinzaine d'années, quand les villageois n'avaient encore ni cultures pérennes ni systèmes d'irrigation sur pentes.

Après trois tours (années) de jeu, les participants sont invités à discuter des problèmes soulevés dans le jeu et à proposer des solutions pour y remédier. Puis les représentants du village au conseil du TAO (eux-mêmes participants) soumettent la proposition à la présidente du conseil pour discussion. Enfin, en adaptant les règles du jeu, la proposition collectivement négociée est testée lors des trois tours de jeu suivants.

rôles sont présentés dans l'encadré 1 . Ce jeu est joué par 12 agriculteurs du village, nombre correspondant à un équilibre entre les contraintes pratiques d'animation d'un tel jeu qui doit rester fluide et ludique et la nécessité d'avoir suffisamment de joueurs pour stimuler un processus d'interactions intéressant. La sélection des 12 participants reposait sur une connaissance fine du contexte socio-économique et politique local et fut établie en concertation avec l'institutrice du village. Les participants choisis représentaient la diversité des intérêts existant sur la question de l'eau. Sur les 12 participants, 3, 6 et 3 agriculteurs (hommes ou femmes) géraient des exploitations de types $\mathrm{A}, \mathrm{B}$ et $\mathrm{C}$ ( $c f$. tableau 1) respectivement, ce qui correspondait à la répartition observée dans le village. Les participants avaient dans le jeu un statut socio-économique (types A, $\mathrm{B}$ ou $\mathrm{C}$ ) proche de la réalité.

Le modèle SMA est très proche du jeu de rôles : aux 12 joueurs correspondent 12 agents « agriculteurs », au plateau de jeu correspond l'interface spatiale du SMA, au tour de jeu correspond le pas de temps de la simulation, etc. (Barnaud et al, sous presse). À chaque pas de temps, les agents du modèle prennent la même série de décisions que les joueurs, avec les mêmes contraintes, la différence majeure étant que dans le jeu, ce sont des joueurs réels qui prennent la décision, tandis que dans le modèle ces processus décisionnels sont modélisés et simulés.

Atelier participatif combinant sessions de jeu de rôles et simulations SMA

Le jeu de rôles et le modèle SMA ont été utilisés lors d'un atelier participatif de trois jours dans l'école du village (figure 3).

Le premier jour fut consacré aux sessions de jeu de rôles :

- première session de jeu pour mise en situation des participants et mise en évidence des problèmes (figure $3 a$ ) ;

- discussion collective sur les problèmes 
Figure 3. Atelier participatif combinant jeu de rôles et simulations multi-agents

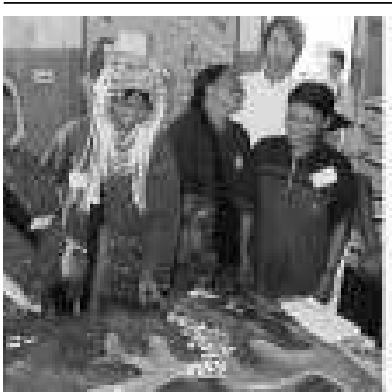

Figure 3a. Joueurs discutant autour du plateau de jeu pendant une session de jeu

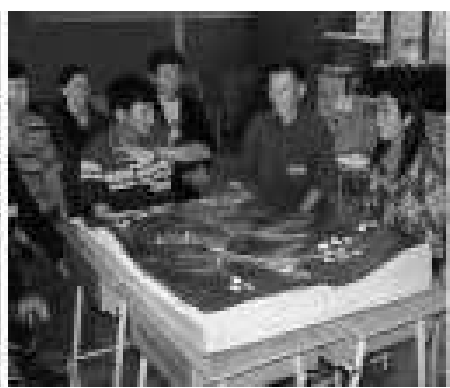

Figure $3 b$. Présentation des propositions des villageois à la présidente de l'administration du TAO

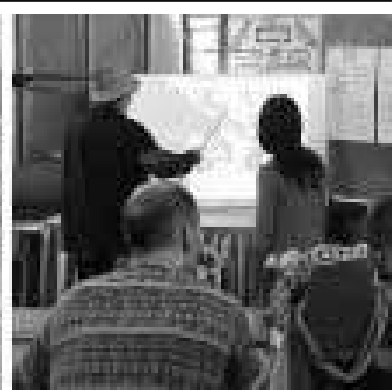

Figure $3 c$. Simulations participatives à l'aide du modèle SMA

Crédit photos : les auteurs identifiés et les solutions envisagées ; - présentation des propositions à la présidente du TAO pour discussion (figure $3 b$ );

- deuxième session pour tester une solution proposée.

Le deuxième jour fut consacré à des interviews individuelles auprès des joueurs pour : - évaluer l'adéquation entre leur représentation du problème et celle des chercheurs ;

- mieux comprendre leurs comportements et décisions au cours du jeu,

- connaître leur avis personnel sur les sujets collectivement débattus.

C'est également ce jour-là que les critiques des joueurs sur le modèle du jeu ont été prises en compte pour ajuster le modèle SMA aux perceptions des joueurs.

Le troisième jour de cet atelier fut consacré aux simulations participatives à l'aide du modèle SMA (figure 3c) : (i) scénario représentant la situation actuelle posant problème, (ii) scénarios proposés par les joueurs pour résoudre le problème.

Des simulations particulières faisant participer activement les 12 villageois ont été réalisées à l'aide d'un modèle hybride dans lequel les 12 agents informatiques du modèle prenaient des décisions concernant toutes les activités (crédit, assolement, etc.) sauf l'allocation de l'eau. Avec ce modèle hybride, la simulation s'arrête au moment de prendre les décisions concernant l'irrigation. Les 12 participants qui suivent l'évolution de « leur » agent informatique à l'écran doivent alors décider collectivement l'allocation des ressources en eau disponibles (lesquelles varient en fonction de la pluviométrie). La répartition de la ressource décidée est alors entrée dans le modèle avant de poursuivre la simulation.

Dans cette expérience, aucune réunion formelle ne fut organisée suite au jeu pour restituer le processus à la communauté dans son ensemble. Mais les ateliers ont stimulé de nombreuses discussions au village, et c'est par le biais de ces échanges spontanés et informels que l'information a circulé entre les participants et les nonparticipants. C'est ainsi que des villageois qui n'avaient jamais participé aux ateliers sont venus nous trouver pour exprimer leur souhait de participer aux ateliers suivants. Cependant, comme les villageois l'expriment eux-mêmes, il reste une différence entre «ceux qui ont joué » et «ceux n'ont pas joué »; c'est une voie d'amélioration de la méthode discutée dans Barnaud et al. (2006b).

\section{Suivi-évaluation des effets du processus ComMod}

De nouvelles interviews individuelles ont été menées trois semaines puis trois mois après 
l'atelier pour en évaluer les effets. Par ailleurs, de nouvelles simulations participatives avec le modèle hybride furent conduites au sein de petits groupes socialement homogènes cette fois pour accompagner l'évolution de la réflexion collective sur les scénarios de nouvelles règles d'allocation de l'eau.

\section{Facilitation d'un apprentissage collectif - Résultats et discussion}

\section{Cadre d'évaluation}

Nous présentons et discutons les résultats de ce cycle ComMod au moyen d'une adaptation du cadre méthodologique proposé par Leeuwis et Van Den Ban (op. cit.) décrivant la mise en œuvre de processus d'apprentissage collectif, de négociation et d'action coordonnée. Ces auteurs identifient sept tâches distinctes : (1) préparation (enquêtes, sélection des participants), (2) accord entre partenaires sur les étapes du processus, (3) échange de perceptions sur la situation, (4) identification de solutions, (5) établissement d'un accord, (6) communication avec les institutions locales, (7) action coordonnée. Van Paassen (2004) souligne la nécessité d'évaluer continuellement les effets de la démarche et de l'ajuster en conséquence.

\section{Analyse du processus d'apprentissage collectif stimulé}

Co-construction de la représentation $d u$ système agraire

Selon Lavigne-Delville (2000) le manque de compréhension des contextes locaux est à l'origine de nombre d'échecs d'approches participatives. Ici, suite à une première analyse du système agraire à dire d'experts, la compréhension de la situation n'a cessé d'évoluer au cours du processus par la confrontation continue des représentations des chercheurs et des autres acteurs. Dans la phase de préparation de chacun des cycles, de nouvelles enquêtes ont été menées pour adapter le modèle et le jeu de rôles à leurs représentations et préoccupations. Nous avons ainsi produit non pas un modèle définitif, mais une famille de modèles, chacun intégrant les dynamiques pertinentes pour faciliter des discussions sur une question donnée à un moment donné du processus.

\section{Prise de conscience : un problème \\ à résoudre collectivement}

Selon Röling et al. (op. cit.), la prise de conscience de l'existence d'un problème et de la nécessité de le résoudre collectivement est une étape nécessaire à l'implication des participants dans un processus d'apprentissage collectif. À Mae Salaep, la première session de jeu a mis en évidence les tensions sur l'eau. Dès le premier tour de jeu, deux exploitants aisés ont investi dans des plantations de litchis et de thé Oolong et installé des prises d'eau dans les deux ruisseaux du plateau de jeu en faisant valoir la règle $\mathrm{du}$ « premier arrivé, premier servi ». Cette session de jeu a aussi mis en évidence un groupe d'exploitations aux parcelles localisées au-dessus des ruisseaux qui n'ont aucune possibilité d'irrigation par gravité.

«Le jeu a permis aux joueurs de comprendre par eux-mêmes qu'il est nécessaire de changer les règles actuelles sans que quelqu'un ait à le leur dire » déclara l'un des leaders villageois. Sa remarque souligne l'importance de l'apprentissage expérimental (Kolb, 1984) ${ }^{5}$, stimulé par cet outil qui permet aux joueurs d'observer l'impact de leurs actions et de celles des autres. Leeuwis et Van Den Ban (op. cit.) distinguent les retours positifs d'information (indiquant au participant qu'il est sur la bonne voie) des retours négatifs (indiquant l'existence d'un problème et stimulant la décision d'agir pour le résoudre). Ces deux types de retours ont été combinés ici.

5. In Leeuwis et Van Den Ban (2004). 
À ce stade du processus d'apprentissage collectif, on ne peut pas dire qu'il y ait eu un gain réel de connaissances. Mais cette mise en évidence collective du problème était une étape préalable nécessaire pour en discuter. En effet, sans cette session de jeu, il est très probable que les agriculteurs les plus puissants qui ont accès à l'eau et n'ont pas intérêt à ce que cette question soit débattue l'auraient fait passée sous silence.

\section{Échange de perceptions sur le problème et identification collective de solutions}

À la fin de la première session de jeu, chacun s'exprima sur les problèmes rencontrés. Un participant (exploitant aisé et représentant du village au TAO) prit le rôle de porte-parole et résuma ainsi la situation : «Il y a deux problèmes à résoudre: le manque d'eau et l'impossible accès à l'eau pour les gens dont les terres sont au-dessus des ruisseaux. » Il est intéressant de remarquer que cette formulation du problème ne met pas directement en cause la règle d'allocation de l'eau en vigueur. Nous verrons par la suite qu'elle ne pouvait être abordée frontalement.

Les participants furent alors invités à discuter de possibles solutions. Le représentant au TAO proposa d'abord la construction d'un imposant réservoir au-dessus du village sensé pourvoir de l'eau à tous, proposition accueillie avec scepticisme par les autres joueurs car une telle solution ne bénéficierait en fait qu'à une minorité d'exploitations dont les parcelles étaient situées sur un flanc du bassin-versant. Un autre joueur (leader de la communauté chrétienne du village) proposa lui d'aménager une retenue collinaire sur chaque ruisseau du bassinversant dont l'eau stockée serait partagée entre 4 ou 5 agriculteurs. Tous les joueurs acceptèrent cette idée, sauf l'un d'eux dont les parcelles sont au-dessus des ruisseaux. Après de nouvelles négociations, le représentant au TAO fit la synthèse en reprenant l'idée des petits barrages, mais sans évoquer le problème de ceux qui ne pourraient en bénéficier. Comment les participants ont-ils perçu cette phase d'échanges ? Selon eux, l'un des principaux intérêts du jeu réside dans la possibilité de mieux comprendre les perceptions des autres : «Nous avons réalisé que nous étions plusieurs concernés par le même problème. Dans la réalité, chacun va tous les jours dans ses champs et nous n'avons pas de telles occasions d'échanges » dit un joueur. Ils évoquent également le fait que la recherche collective de solutions est plus fructueuse que la réflexion individuelle. Enfin, le jeu et l'ambiance ludique, apaisant les tensions, permettent de : «Parler plus facilement que dans la réalité, même si l'on parle quand même de la réalité », précisa l'un d'eux.

En matière de gestion des ressources communes, Ostrom (op. cit.) distingue les problèmes de disponibilité (quantité de ressource disponible) des problèmes d'appropriation (partage de la ressource). Il est intéressant de noter qu'ici la question délicate de l'appropriation n'est abordée qu'à travers celle de la disponibilité. Selon le leader chrétien du village : "La seule façon de changer les règles c'est de construire un aménagement collectif obligeant les gens à discuter et à mettre en place de nouvelles règles, comme cela s'est passé dans le jeu. Sans nouvel aménagement, les règles ne changeront pas. » L'analyse des relations de pouvoir dans ce processus permet de mieux comprendre cette phase de l'apprentissage collectif. Il était impossible pour les petits agriculteurs n'ayant pas accès à l'eau d'irrigation de remettre en cause directement la règle en vigueur car les agriculteurs les plus puissants ayant accès à l'eau auraient simplement refusé de participer à la négociation. L'habileté de la proposition du leader chrétien réside dans le fait qu'il ne suggère pas de remettre directement en question la façon dont « le gâteau est partagé », mais de réfléchir collectivement à la façon dont les villageois pourraient « augmenter la taille du gâteau ». Il s'agit d'un processus de négociation dit créatif 
ou intégratif (Leeuwis et Van Den Ban, 2004).

\section{Exploration de scénarios avec jeu de rôles et simulations}

Dans l'apprentissage, Leeuwis et Van Den Ban (2004) distinguent l'échange de perceptions entre les gens de l'amélioration de la compréhension de la situation. Si le premier domine dans la phase de négociation d'une solution, l'évaluation de ses effets relève de la seconde. Avec ComMod, cette exploration du système passe par la simulation de scénarios, à l'aide du jeu de rôles et du modèle SMA. Dans notre cas, le test dans le jeu de la proposition « petites retenues collinaires » a soulevé de nouvelles discussions sur les règles de partage de l'eau entre bénéficiaires. Les séances de simulations participatives en petits groupes à l'aide du modèle SMA hybride ont permis d'appuyer ces négociations. La discussion des résultats de ces simulations fit émerger deux règles de partage possibles de l'eau :

- proportionnel aux surfaces à irriguer ; - égalitaire de la ressource, les exploitations ayant un excédent d'eau prêtant temporairement leurs droits aux exploitations déficitaires.

Les simulations ont mis en évidence un creusement plus important des écarts de revenus dans le cas du scénario «partage proportionnel » par rapport à l'autre.

Ostrom (1994) souligne la nécessité pour les usagers de définir eux-mêmes les règles d'accès à la ressource et ce processus a été initié lors de cette expérience. «S'il y avait aménagement d'une retenue collective, il faudrait se mettre d'accord dès le début sur les règles de partage de l'eau. Si elles sont bien établies, tous les gens les respecteront, sans exception » dit un aîné. "Il faudra mettre en place un responsable qui règle les litiges » ajouta un autre joueur. Les simulations ont facilité l'identification et la négociation de telles règles en toute connaissance de causes.

L'évolution des règles proposées par les villageois fut particulièrement intéressante. Dans un premier temps, au troisième jour de l'atelier, le représentant au TAO (agriculteur aisé gérant de grandes plantations irriguées) imposa sans réelle discussion possible la règle d'un partage de l'eau proportionnel aux surfaces plantées. Trois semaines plus tard, les villageois continuaient à en discuter entre eux et s'étaient mis d'accord : si de telles retenues collinaires étaient construites, il faudrait mettre en place un partage égalitaire de l'eau. Nous reviendrons sur l'analyse de cette évolution à la lumière des enjeux de pouvoir.

\section{Voies d'amélioration de la méthode}

Une attention aux conflits d'intérêts

Un joueur disait voir le jeu comme un espace démocratique où toutes les voix pouvaient s'exprimer. Est-ce réellement le cas ? Et ont-elles été prises en compte? Ainsi, le cas des exploitants dont les parcelles seraient au-dessus des retenues collinaires a été éludé au fil des discussions. L'un d'eux dit plus tard que si cela arrivait, « [il] ne dirait rien, [il] subirait». Ceci illustre la difficulté de trouver une solution unique et consensuelle dans ces milieux socialement et écologiquement très hétérogènes. Mermet (2005) écrit que dans un processus de négociation, il faut donner les moyens à chacun de comprendre les enjeux de la négociation, de s'exprimer et d'avoir un poids dans la discussion. C'est un défi à Mae Salaep où il y a un monde entre le représentant au TAO qui parle la langue thaïe et joue un rôle politique local et ceux qui ne parlent que la langue akha et n'ont jamais quitté le village. Cela implique de renforcer la confiance qu'ont les participants en leur capacité à participer aux négociations. L'évolution de leur attitude au cours des cycles successifs démontre que le processus ComMod a en partie atteint cet objectif. Les enquêtes individuelles, au cours desquelles les participants purent s'exprimer 
hors de portée de certaines influences, furent également déterminantes. Finalement, les effets des jeux de pouvoir et d'influence ont été rendus plus transparents. Van der Veen (2000) insiste sur la phase de formulation d'une proposition car il y a un risque que les plus influents, ou les plus intelligents, imposent - volontairement ou non leur solution. Ce fut le cas notamment lorsque le représentant au conseil du TAO tenta d'imposer son idée de partage de l'eau proportionnel aux surfaces lors des premières séances de simulation. Van Paassen (2004) parle de l'importance des discussions qui ont lieu en coulisses, ce que confirme l'évolution des règles proposées par les autres participants lors des séances de simulations ultérieures. Le processus ComMod a non seulement contribué à rendre les enjeux de pouvoir plus transparents, mais il a participé au renforcement de la voix des plus faibles dans le processus de prise de décision collective, et ce à l'aide de deux mécanismes : un renforcement des capacités individuelles des acteurs les plus faibles à s'exprimer et, au niveau collectif, la création d'une coalition entre le leader chrétien et les agriculteurs les plus pauvres du village augmentant le pouvoir de négociation de ces derniers (Barnaud et al, 2006b). La façon dont ComMod s'introduit ainsi dans les jeux de pouvoir pose la question du risque que le jeu soit utilisé par certains acteurs pour renforcer leur influence, ou au contraire que le renforcement de la voix des moins influents vienne bouleverser un ordre, certes inégal, mais pacifique, valeur fondamentale de la culture akha. Ces risques soulignent la nécessité de bien connaître le contexte sociopolitique local, pour éviter le creusement des inégalités, ainsi que de mettre en place un suivi-évaluation des effets sur la communauté de ce type de démarche. Cela implique aussi de la part de l'animateur d'expliciter ses objectifs (l'équité en est un), afin de pouvoir le remettre en cause et d'en discuter ouvertement la légitimité avec les différentes parties prenantes. L'idée sous-jacente est que l'animateur neutre n'existe pas et qu'une telle neutralité proclamée présenterait des risques de manipulation insidieux, car non explicités et donc plus difficiles à remettre en cause.

\section{Renforcer le dialogue \\ avec les administrations locales}

La rencontre entre les villageois et la présidente du conseil du TAO ne s'est pas soldée par le dialogue « ascendant » escompté : elle a balayé leur proposition d'une phrase avant de se lancer, en langue thaïe (bien qu'elle parle aussi akha), dans une tirade sur les mesures environnementales discutées à Bangkok. Les participants ont tout de même décidé suite au jeu de se concerter avec les autres villageois pour rédiger une demande officielle de financement au TAO. Dix mois plus tard, ils avaient rédigé et soumis un projet de petits barrages sur les trois sous bassins versants du village. Concernant les règles d'allocation de l'eau, l'un des leaders villageois précisait que lorsque le projet serait accepté, de nouvelles sessions de jeu seraient utiles pour discuter collectivement des règles d'allocation de l'eau à mettre en place. En 2006, la demande de financement des villageois n'avait malheureusement pas été retenue par la présidente du TAO, mais cette dernière encourageait les villageois à soumettre à nouveau leur projet ultérieurement. Par ailleurs, forts de leur projet commun, les villageois commençaient à évoquer d'autres sources possibles de financement.

Une meilleure coordination interinstitutionnelle est donc nécessaire, non seulement pour espérer un soutien financier, technique et institutionnel des projets villageois, mais également pour accroître la marge de manœuvre des communautés par rapport aux niveaux d'organisation supérieurs. La tradition de rapports autoritaires entre organismes de développement et communautés freine ici l'établissement de ce dialogue, la difficulté venant des communautés non habituées à prendre l'initiative, mais aussi des 
agents des organisations dont les discours évoluent plus vite que les mentalités. Si cette expérience ComMod a réussi à vaincre la première difficulté, la seconde reste entière. Parmi les sept tâches constitutives d'un apprentissage collectif identifiées dans le paragraphe relatif au cadre d'évaluation, les objectifs des tâches (1) préparation, (3) échange de perceptions et (4) identification de solutions, ont été atteints, mais un effort reste à fournir pour les autres tâches qui concernent le dialogue avec les institutions aux niveaux supérieurs. Trois pistes d'amélioration peuvent être envisagées. Leeuwis et Van Den Ban (2004) mentionnent l'importance de la définition précoce des objectifs du processus, de ses étapes et des résultats attendus avec les institutions impliquées, ce qui ne fut pas réalisé à Mae Salaep où le diagnostic initial ne s'attarda pas sur l'analyse institutionnelle. Van Paassen (2004) suggère aussi qu'après la prise de contact avec ces organisations, la négociation de solutions avec elles ne prenne place qu'une fois que les acteurs locaux sont en confiance et ont forgé un solide accord entre eux. Enfin, une plus forte implication d'un acteur local facilitant le processus ComMod favoriserait un contact suivi entre les diverses institutions en présence, nous revenons sur ce point dans la section suivante.

\section{Impliquer un facilitateur local}

Olsson et al. (2004) ont analysé les transformations institutionnelles favorables à l'émergence d'une gestion collective d'un écosystème et soulignent le rôle clef joué par certains acteurs locaux moteurs dans le processus. Un tel facilitateur procurerait plus de légitimité, de continuité et un impact accru du processus ComMod. À Mae Salaep, l'implication des agents du « Department of Public Welfare » est jusqu'à présent restée de l'ordre du soutien logistique, les chercheurs restant moteurs du processus. Pour qu'un facilitateur local puisse un jour piloter le processus, Van Paassen (op. cit.) mentionne la nécessité d'identifier une personne dont la mentalité est en accord avec l'esprit du projet. Leeuwis (op. cit.) quant à lui insiste sur la définition précoce avec les facilitateurs des objectifs de la démarche et de ce qui est attendu d'eux, tout en vérifiant que ces attentes soient compatibles avec leurs capacités, agendas et leurs marges de manœuvre, ce qui est très pertinent dans la bureaucratie thaillandaise hautement hiérarchique. C'est une des raisons pour lesquelles les démarches participatives au nord de la Thaïlande se sont jusqu'à présent surtout adressées aux $\mathrm{ONG}$ plutôt qu'aux agences gouvernementales. Mais c'est justement l'absence de lien avec ces dernières institutions qui en a limité l'impact.

\section{Conclusion}

À partir d'une étude de cas, cet article explore les apports et limites de la modélisation d'accompagnement pour faciliter une gestion concertée et décentralisée des ressources au nord de la Thaillande. Actuellement, l'un des maillons faibles de la décentralisation est le manque de dialogue entre les communautés et les administrations locales comme les TAO. Un tel dialogue implique un double effort. D'une part il faut donner les moyens aux communautés de saisir la complexité des problèmes examinés, d'en cerner les enjeux sociaux et écologiques et de faire des propositions d'amélioration adaptées. Étant donné les divergences d'intérêts entre les divers types d'agriculteurs d'une communauté, cela implique la mise en œuvre non seulement d'un processus d'apprentissage collectif, mais également de négociation au sein de la communauté villageoise. D'autre part il s'agit de leur permettre de défendre leurs propositions auprès des institutions gouvernementales comme les TAO afin qu'elles soient prises en compte dans l'affectation de leurs moyens budgétaires. L'expérience de Mae Salaep montre que ComMod accompagne bien les processus d'apprentissage 
collectif et de négociation au niveau des communautés. Le processus a facilité la construction collective d'une représentation de la situation, la prise de conscience de l'existence d'un problème commun et de la nécessité de le résoudre collectivement. Il a aussi stimulé un échange de perceptions sur ce problème et la négociation de solutions possibles en s'aidant de l'exploration de scénarios mettant en avant les impacts sociaux et écologiques des alternatives proposées ainsi que leurs effets sur les différentes catégories socio-économiques d'exploitations. Dans des contextes socialement hétérogènes, une attention soutenue aux conflits d'intérêts et aux jeux de pouvoir entre les membres de la communauté est nécessaire pour éviter que la démarche ne se solde par un creusement des inégalités existantes.

Mais la démarche doit être améliorée pour ce qui concerne la prise en compte des propositions villageoises par les institutions aux niveaux supérieurs. Nos recommandations à ce sujet se situent à deux niveaux. Tout d'abord, indépendamment de la démarche elle-même, il existe un frein propre au contexte thaïlandais dans lequel décentralisation ne rime avec participation que sur le papier. Dans la pratique, les mentalités de la plupart des fonctionnaires des institutions décentralisées évoluent lentement. Il est donc nécessaire de former une génération d'agents publics maîtrisant les démarches de gestion participative des ressources. D'autre part, l'amélioration de la démarche ComMod elle-même est nécessaire afin d'augmenter les chances d'établir un dialogue fructueux avec les institutions aux niveaux supérieurs. Il faut pour cela identifier dès le début du processus les organisations pouvant être impliquées ainsi que les personnes ouvertes aux approches participatives en leur sein afin de construire une relation de confiance avec elles, de mieux comprendre leurs intérêts et d'établir en vue de quels objectifs et avec quelles marges de manœuvre ces institutions seraient intéressées à s'impliquer.

Un renforcement du dialogue avec les institutions aux niveaux supérieurs permettrait en retour une extension géographique horizontale de l'impact de la démarche, les marges de manœuvre négociées par une communauté pouvant par la suite faciliter les initiatives d'autres communautés. Par ailleurs, une fois sélectionnés et formés, les facilitateurs locaux pourraient aussi transmettre leur savoir-faire. Enfin, le problème du coût de ce type de processus de concertation n'en serait plus un si une telle démarche prenait place dans le programme de travail habituel des organismes de gestion des ressources, en remplacement, par exemple, d'une partie de leurs nombreuses réunions classiques. 


\section{RÉFÉRENCES BIBLIOGRAPHIQUES}

Arghiros D. (2001). Democracy, Development and Decentralization in Provincial Thailand. Curzon \& Nordic Institute of Asian Studies, Richmond, Surrey, 308 p.

Barnaud C., Promburom P., Bousquet F., Trébuil G. (2006a). Companion Modelling to Facilitate Collective Land Management by Akha Villagers in Upper Northern Thailand. Journal of World Association of Soil and Water Conservation, $\mathrm{n}^{\circ} 1$, p. 38-54.

Barnaud C., Van Paassen A., Trébuil G. (2006b). Power Relations and Participatory Water Management: lessons from a Companion Modeling experiment in a highland community of Northern Thailand. Communication présentée au colloque international "International Forum on Water and Food", 12-17 novembre, Vientiane, Laos.

Barnaud C., Promburom T., Trébuil G., Bousquet F. (2007). Evolving Simulation and Gaming to Support Adaptive Watershed Management in Mountainous Northern Thailand. Journal Simulation and Gaming, $\mathrm{n}^{\circ} 38$, p. 398-420

Barnaud C., Bousquet F., Trébuil G. MultiAgent Simulations to Explore Rules for Rural Credit in a Highland Farming Community of Northern Thailand. Ecological Economics, $n^{\circ}$ 66(4), p. 615-627.

Barreteau O., Bousquet F., Attonaty J. (2001). Role-playing games for opening the black box of multi-agent systems: method and lessons of its application to Senegal River valley irrigated systems. Journal of Artificial Societies and Social Simulation 4(2). http://www.soc.surrey.ac.uk/JASSS/4/2/5. html

Berkes F., George P., Preston R. (1991). Co-management: the evolution of the theory and practice of joint administration of living resources. Alternatives 18 (2), p. 12-18.

Bousquet F., Barreteau O., Mullon C., Weber J. (1996). Modélisation d'accompagne- ment : systèmes multi-agents et gestion des ressources renouvelables. In «Quel environnement au XXI siècle? Environnement, maîtrise du long terme et démocratie » Abbaye de Frontevraud.

Bousquet F., Cambier C., Morand P., Quensière J., Mullon C., Pavé A. (1993). Simulating the interaction between a society and a renewable resource. Journal of Biological Systems, $\mathrm{n}^{\circ} 1$ (1), p. 199-214.

Bousquet F., Trébuil G. (2005). Introduction to companion modeling and multi agent systems for integrated natural resource management in Asia. In Bousquet F., Trébuil G., Hardy (Eds), "Companion Modeling and Multi-Agent Systems for Integrated Natural Resource Management in Asia", Los Baños, International Rice Research Institute, Philippines, p. 1-17. Carlsson L., Berkes F. (2004). Co-management: concepts and methodological implications. Journal of Environmental Management 75, p. 65-76.

Commod C. (2005). La modélisation comme outil d'accompagnement. Natures, Sciences Sociétés, ${ }^{\circ} 13$, p. 165-168.

D'Aquino P., Le Page C., Bousquet F., Bah A. (2003). Using Self-Designed Role-Playing Games and a Multi-Agent System to Empower a Local DecisionMaking Process for Land Use Management: The SelfCormas Experiment in Senegal. Journal of Artificial Societies and Social Simulation 6(3), http://jasss.soc.surrey.ac.uk/6/3/5.html Étienne M. (2003). Sylvopast: a multiple target role playing game to assess negociation processes in sylvopastoral management planning. Journal of Artificial Societies and Social Simulation 6(2), http://jass.soc.surrey.ac.uk/6/2/5.html Ferber J. (1999). Multi-Agent Systems: An Introduction to Distributed Artificial Intelligence. Boston, Addison-Wesley Longman. 
Funtowicz S.-O., Ravetz J.-R. (1994). The worth of a songbird: ecological economics as a post-normal science. Ecological Economics, ${ }^{\circ} 10$ (3), p. 197-207.

Ganjanapan A. (2002). Complexity of rights and legal pluralism in participatory watershed management. In Jianchu and Mikesell (Eds), "Yunnan Science and Technology Press, Lanscapes of diversity", Proceedings of $3^{\text {rd }}$ International Conference on Montane Mainland Southeast Asia (MMSEA 3), Lijiang, China, p. 207-212.

Goodman J. (1996). Meet the Akhas. White Lotus, Bangkok.

Gurung T.-R., Bousquet F., Trébuil G. (2006). Companion modeling, conflict resolution, and institution building: sharing irrigation water in the Lingmuteychu Watershed, Bhutan. Ecology and Society 11(2), p. 36, http://www.ecologyandsociety.org/vol11/iss2/art36/

Hardin G. (1968). The Tragedy of the Commons. Science, ${ }^{\circ} 162$, p. 1243-1248.

Holling C.-S. (2001). Understanding the complexity of economic, ecological and social systems. Ecosystems, $\mathrm{n}^{\circ} 4$, p.390-405.

Lansing J.-S., Kremer J.-N. (1993). Emergent properties of Balinese water temple networks: coadaptation on a rugged fitness landscape. American Anthropologist 95(1), p. 97-114.

Lavigne-Delville P., Selamna N.-E., Mathieu M. (2000). Les enquêtes participatives en débat, Ambition, pratiques et enjeux. Paris, Karthala, ICRA, GRET, 543 p.

Le Meur P.-Y. (2000). Les hautes terres du Nord en Transition. Développement, courtage et construction nationale. Revue Tiers Monde, $\mathrm{n}^{\circ}$ XVI (162).

Leeuwis C., Van Den Ban A.-W. (2004). Communication for rural innovation. Rethinking agricultural extension. Blackwell publishing Ltd, Oxford.

McKinnon J., Vienne B. (1989). Hill tribes today. White Lotus-Orstom, Bangkok, $507 \mathrm{p}$.
Mermet L. (2005). Concertations orchestrées ou négociations décisives? Programme Concertation, Décision et Environnement, Tome I, ministère de l'Écologie et du Développement durable.

Mermet L. (1993). La nature comme jeu de société. Paris, L'Harmattan.

Neef A. (2004). People's participation in natural resource management in northern Thailand - Paradgm shift or old wine in new bottles? Communication presented at $4^{\text {th }}$ EUROSEAS Conference, Paris, 2004.

Ollagnon H. (1989). Une approche patrimoniale de la qualité du milieu naturel. In Mathieu N. et Jollivet M. (Eds) «Du rural à l'environnement, la question de la nature aujourd'hui », Paris, L'Harmattan, p. 258-268.

Olsson P., Folke C., Hahn T. (2004). Socialecological transformation for ecosystem management: the development of adaptive co-management of a wetland landscape in southern Sweden. Ecology and Society, $\mathrm{n}^{\circ} 9$ (4).

Ostrom E. (2005). Understanding institutionnal diversity. New Jersey, Princeton University Press.

Ostrom E., Gardner R., Walker J. (1994). Rules, games \& common-pool resources. University of Michigan Press, Michigan, USA.

Pungprasert V. (1989). Hill tribe people blamed for deforestation. In McKinnon J. et Vienne B. (Eds), Hill tribes today, Golden Lotus, Bangkok, p. 363-367.

Rerkasem K., Rerkasem B. (1994). Shifting cultivation in Thailand: its current situation and dynamics in the context of Highland Development. London, International Institute for Environment and development, $140 \mathrm{p}$.

Rola A.-C., Coxhead I. (2005). Economic development and environmental management in the uplands of Southeast Asia: challenges for policy and institutionnal development. Agricultural economics, n 32 (1), p. 243-256. 
Röling N.-G., Wagemakers M.-A. (1998). A new practise: facilitating sustainable agriculture. In Röling N.-G., Wagemakers (Eds), "Facilitating Sustainable Agriculture: Participatory learning and adaptive management in times of environmental uncertainty", Cambridge, Cambridge University Press, p. 3-22.

Rutherford J. (2002). Institutions, Impacts and responses in the agrarian transformation of the mountains of northern Thailand. In Jianchu and Mikesell (Eds.), "Yunnan Science and Technology Press, Lanscapes of diversity". Proceedings of the $3^{\text {rd }}$ International Conference on Montane Mainland Southeast Asia (MMSEA 3), Lijiang, China, p. 55-78.

Thomas D.-E., Weyerhaeuser H., Sapathong P. (2002). Improved tools for managing agroforestry landscapes in Northern thailand: pilot application of spatial analysis and negociation support systems. In Jianchu and Mikesell (Eds.), "Yunnan Science and Technology Press, Lanscapes of diversity". Proceedings of the $3^{\text {rd }}$ International Conference on Montane Mainland Southeast Asia (MMSEA 3), Lijiang, Yunnan, China, p. 381-400.

Trébuil G. (1993). Agriculture pionnière, révolution verte et dégradation de l'environnement en Thaïlande : le cinquième dragon ne sera pas vert, $\mathrm{PUF}$ et IEDES, Université PanthéonSorbonne, Paris, 1993 et Tiers-Monde, $\mathrm{n}^{\circ} 134$, p. $365-383$.

Trébuil G., Kam S.-P., Turkelboom F., Shinawatra B. (1997). Diagnoses at Field, Farm and Watershed Levels in Diversifying Upland Agroecosystems: Towards Comprehensive Solutions to Farmers'
Problems. In Kropff, Teng, Aggarwal, Bouma, Bouman, Jones and Laar (Eds.), Kluwer Academic Publishers, "Systems Approaches for Sustainable Agricultural Development : Applications of Systems Approaches at the Farm and Regional Levels". Proceedings from International Rice Research Institute (IRRI) International Symposium, p. 99-114.

Trébuil G., Shinawatra-Ekasingh B., Bousquet F., Thong-Ngam C. (2002). Multiagent systems companion modelling for integrated watershed management: a northern Thailand experience. In Jianchu and Mikesell (Eds.), Yunnan Science and Technology Press, China, "Landcapes of diversity". Proceedings of $3^{\text {rd }}$ International Conference on Montane Mainland Southeast Asia (MMSEA 3), Lijiang, China, p. 55-78.

Trébuil G., Thong-Ngam C., Turkelboom F., Grellet G., Kam S.-P. (2000). Trends of Land Use Change and Interpretation of Impacts in the Mae Chan Area of Northern Thailand. Communication presented at $2^{\text {nd }}$ symposium on Montane Mainland Southeast Asia, "Governance in the Natural and Cultural Landscape", Chiang Mai, Thailand.

Van der Veen, R. G. W. (2000). Learning natural resource management. In Guijt, Berdegué et Loevinshon (Eds), ISNAR and RIMISP, "Deepening the basis of rural resource management", The Hagues, Netherlands, p. 15-21.

Van Paassen A. (2004). Bridging the gap: computer model enhanced learning about natural resource management in Burkina Faso. Wageningen University and Research Center. 